\section{Rapid learning of passive avoidance by weanling rats: Conditioned taste aversion*}

\author{
FREDERICK W. GROTE, JR. \\ Western Washington State College, Bellingham, Wash. 98225 \\ and \\ ROBERT T. BROWN \\ University of North Carolina at Chapel Hill, Chapel Hill, N.C. 27514
}

Weanling rats were allowed to drink lithium chloride solution, a drug which induces visceral upset, at 22 (conditioning) and 25 (testing) days of age. Intake of lithium chloride in testing was significantly below that in conditioning, indicating that the rats learned not to drink the aversive fluid after one exposure and that weanling rats can rapidly learn to inhibit certain responses.

Weanling rats learn passive avoidance of electric shock more slowly than do mature rats (e.g., Brunner, 1969; Riccio, Rohrbaugh, \& Hodges, 1968), although they are not different in learning of active avoidance of electric shock (Kirby, 1963; Riccio et al, 1968). Even with intense (Feigley \& Spear, 1970) or long-duration (Schulenburg, Riccio, \& Stikes, 1971) shocks, weanling rats take several trials to learn passive avoidance, as opposed to the one-trial learning common in mature rats.

Would weanling rats show rapid acquisition of a passive avoidance which they are "prepared," in Seligman's (1970) terms, or "predisposed," in ethological terms (e.g., Manning, 1967), to learn? Notions such as "preparedness" and "predisposition to learn" suggest that a given species may be more adapted to learn certain kinds of associations than others as a function of its evolutionary history. In the present experiment, weanling rats were conditioned to avoid drinking a lithium chloride solution. Seligman (1970) has summarized a variety of data which indicate that rats may be particularly prepared to associate gustatory stimuli with subsequent aversive visceral consequences. Rats were presented with a lithium chloride solution at 22 days of age (conditioning) and again at 25 days of age (testing). Lithium chloride was chosen as the aversive substance because it has a distinctive salty taste and, although initially palatable to rats, produces visceral upset within a few minutes of ingestion. Adult rats learn not to drink lithium chloride

*This research was partly supported by Grant VF042 from the University of North Carolina Research Council to Robert $T$. Brown. During conduct of this research, Fred W. Grote was a Morehead Graduate Fellow at the University of North Carolina. We thank Mead, Johnson Co. for drugs used in preliminary research. daily 1-h experimental sessions. housed in standard laboratory cages with Purina Lab Chow freely available. PROCEDURE

At the age of 20 days, the rats were placed on a restricted fluid regimen. One of two fluids, water or lithium chloride, was available for $1 \mathrm{~h}$ each morning (experimental session) on the schedule described below. Water was available for $5 \mathrm{~h}$ each evening, beginning $8 \mathrm{~h}$ after the end of the experimental session. Preliminary studies indicated that evening sessions were necessary to provide rats of this age with sufficient water to maintain normal growth.

Conditioning and test sessions were on Days 3 and 6, respectively. A 0.12-M lithium chloride solution was presented in the 1-h period. On Days $1,2,4$, and 5 , water was presented in the experimental session. Drinking during experimental sessions was from $50-\mathrm{ml}$ tubes attached to the front of the home cages. The amount of fluid consumed was determined by weighing the tubes on a chemical balance before and after each session.

RESULTS AND DISCUSSION

The mean intake of fluid in each experimental session is shown in Fig. 1. Lithium chloride intake in the conditioning session did not differ significantly from water intake in Session $2(t=1.75, d f=17, p>.05)$ but was highly significantly lower than water intake in Session $4(t=16.77$, $\mathrm{df}=17, \mathrm{p}<.001)$.

The weanling rats learned not to drink lithium chloride after one

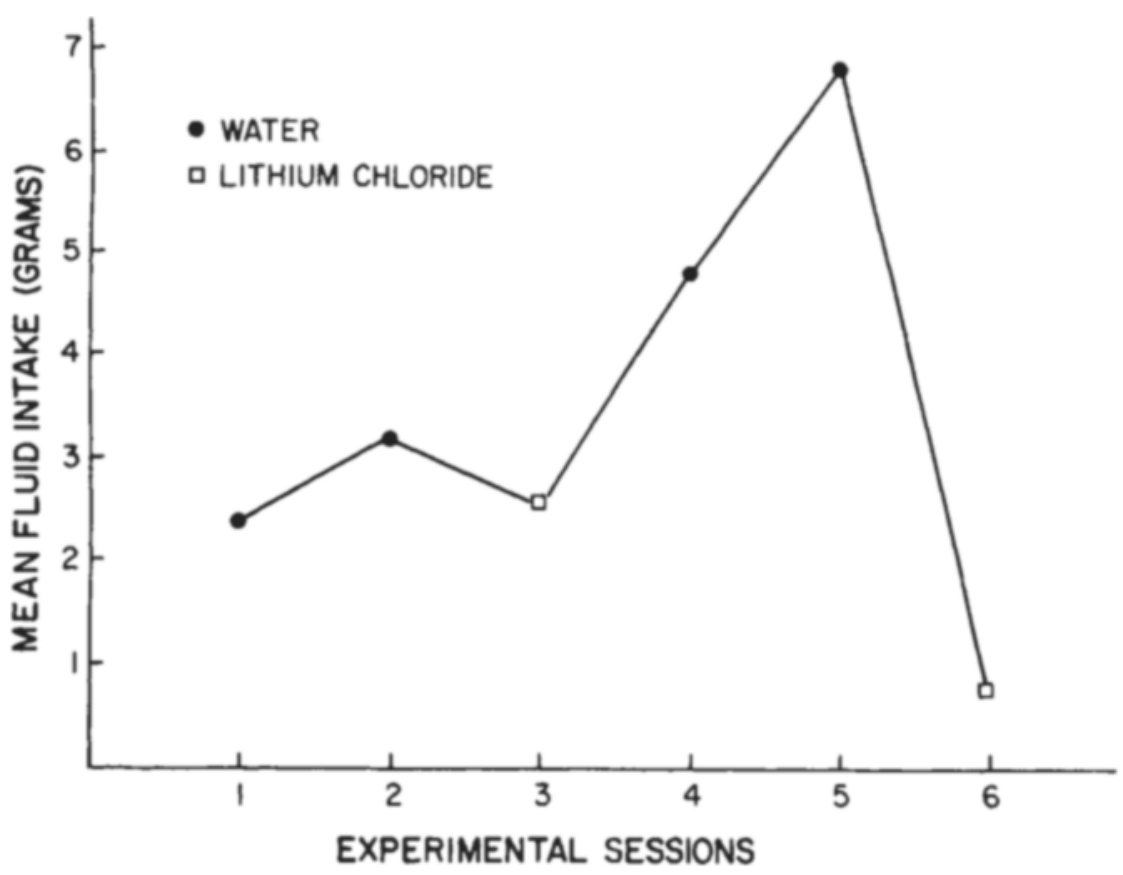

Fig. 1. Mean intake of water and lithium chloride by weanling rats during 
exposure, drinking significantly less lithium chloride in the test session than in the conditioning session $(\mathrm{t}=12.9, \quad \mathrm{df}=17, \quad \mathrm{p}<.001)$. The conditioned aversion was to lithium chloride and not to fluids in general, as indicated by greater water intake in Session 4 than in Session $2 \quad(t=4.9$, $\mathrm{df}=17, \mathrm{p}<.001)$. The group results accurately reflect the intake of individual rats: all 18 rats drank less lithium chloride in the test than in conditioning, and all but three drank more water in Session 4 than in Session 2.

In the test, 10 rats drank 0.5-0.6 g, 4 drank 0.7-0.8 g, 3 drank 0.9-1.0 g, and 1 drank $1.3 \mathrm{~g}$. Other studies (Grote \& Brown, unpublished data) using the same procedure, have found that measured intake under asymptotic learning conditions has varied between 0.5 and $0.8 \mathrm{~g}$ in individual rats and that 0.2 to $0.5 \mathrm{~g}$ may be ascribed to spillage from attaching the bottles to the cages. Most of the weanling rats thus demonstrated virtually complete avoidance of the fluid as far as could be determined under the conditions used. However, since adult rats will stop drinking a fluid to which an aversion has been conditioned after taking only a few licks with a volume of less than 20 microliters (Halpern \& Tapper, 1971), additional learning after one exposure might be detected with a more sensitive measure of intake.

Since the rats drank the fluid that produced illness, many licks were directly associated with the illness. However, Grote (1971) has obtained results similar to those of the present experiment, using a procedure in which there was no overlap between drinking of a fluid and onset of illness.
He intubated lithium chloride solution into the stomachs of weanling and adult rats after they had been allowed to drink saccharin solution. All rats rapidly learned to avoid saccharin, and there were no significant differences between weanling and mature rats.

The present study differed in procedure from passive avoidance of shock studies in several ways. In particular, instead of one locomotor response being followed immediately by a discrete shock, a large number of licks were followed by, and overlapped with, illness. Also, once illness began, it continued for some time whether or not licking continued. The rapid learning of a conditioned taste aversion by weanling rats demonstrated here cannot be directly compared to the comparatively slow learning of a passive avoidance of shock. Using comparable procedures, comparable rates of learning might be found.

It is clear, however, that under certain conditions weanling rats can rapidly learn to inhibit some responses. Since the pioneering demonstration by Campbell and his associates (e.g., Campbell, 1967) that electric shock has equivalent motivational properties in rats of different ages, many developmental studies of learning and retention of avoidance of shock have been performed. Results of the present study suggest that it would now be valuable to study developmental trends in learning of avoidance of stimuli other than shock and involving responses other than locomotion. Physiological accounts of development of response inhibition (Campbell, Lytle, \& Fibiger, 1969; Schulenburg et al, 1971) may have to be able to account for different rates of learning of different types of passive avoidance.

\section{REFERENCES}

BRUNNER, R. L. Age differences in one-trial passive avoidance learning. Psychonomic Science, 1969, 14, 134.

CAMPBELL, B. A. Developmental studies of learning and motivation in infra-primate mammals. In $H$. W. Stevenson, E. H. Hess, and H. L. Rheingold (Eds.), Early behavior: Comparative and developmental approaches. New York: Wiley, 1967.

CAMPBELL, B. A., LYTLE, L. D., \& FIBIGER, H. C. Ontogeny of adrenergic arousal and cholinergic inhibitory mechanisms in the rat. Science, 1969. 166, 635-637.

FEIGLEY, D. A.. \& SPEAR, N. C. Effect of age and punishment condition on long-term retention by the rat of activeand passive-avoidance learning. Journal of Comparative \& Physiological Psychology, $1970,73,515-526$.

GROTE, F.W. JR. Leaming, retention, and extinction of a conditioned aversion to saccharin by weanling and mature rats. Unpublished doctoral dissertation. University of North Carolina, 1971.

HALPERN, B. P. \& TAPPER, D. N. Taste stimuli: Quality coding time. Science. $1971,171,1256-1258$.

KIRBY, R. H. Acquisition, extinction, and retention of an avoidance response in rats as a function of age. Journal of Comparative \& Physiological Psychology, 1963, 56, 158-162.

MANNING, A. An introduction to animal behavior. Reading, Mass: Addison-Wesley, 1967

NACHMAN, M. Learned aversion to the taste of lithium chloride and generalization to other salts. Journal of Comparative \& Physiological Psychology, $1963,56,343-349$.

RICCIO, D. C., ROHRBAUGH, M., \& HODGES, L. A. Developmental aspects of passive and active avoidance learning in rats. Developmental Psychobiology, $1968,1,108-111$.

SCHULENBURG, C. J., RICCIO, D. C., \& STIKES, E. R. Acquisition and retention of a passive-avoidance response as a function of age in rats. Journal of Comparative \& Phy siological Psychology, $1971,74,75-83$.

SELIGMAN, M. E. P. On the generality of the laws of leaming. Psychological Review, 1970, 77, 406-418. 